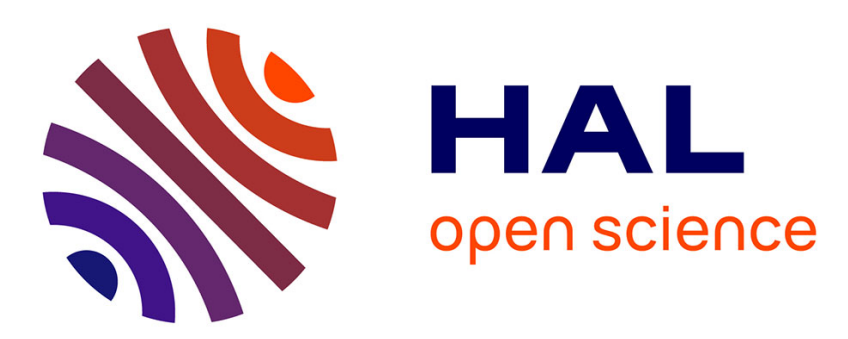

\title{
On the degree of second-order non-circularity of complex random variables
}

\author{
Jean-Pierre Delmas, Habti Abeida
}

\section{To cite this version:}

Jean-Pierre Delmas, Habti Abeida. On the degree of second-order non-circularity of complex random variables. ICASSP 2008: IEEE International Conference on Acoustics, Speech and Signal Processing, Mar 2008, Las Vegas, United States. pp.3905 - 3908, 10.1109/ICASSP.2008.4518507 . hal-01378730

\section{HAL Id: hal-01378730 \\ https://hal.science/hal-01378730}

Submitted on 16 Dec 2020

HAL is a multi-disciplinary open access archive for the deposit and dissemination of scientific research documents, whether they are published or not. The documents may come from teaching and research institutions in France or abroad, or from public or private research centers.
L'archive ouverte pluridisciplinaire HAL, est destinée au dépôt et à la diffusion de documents scientifiques de niveau recherche, publiés ou non, émanant des établissements d'enseignement et de recherche français ou étrangers, des laboratoires publics ou privés. 


\title{
ON THE DEGREE OF SECOND-ORDER NON-CIRCULARITY OF COMPLEX RANDOM VARIABLES
}

\author{
Jean-Pierre Delmas and Habti Abeida \\ GET/INT, Département CITI, UMR-CNRS 5157
}

\begin{abstract}
This paper addresses the degree of second-order non-circularity or impropriety of complex random variables and its purpose is to complement previously available theoretical results. New properties of the non-circularity rate (also called circularity spectrum) are given for scalar and multidimensional complex random variables with a particular attention paid to rectilinear random variables, i.e., with maximum circularity spectrum. Finally, the maximum likelihood estimate of the circularity spectrum in the Gaussian case and asymptotic distribution of this estimate for arbitrary distributions are given.
\end{abstract}

Index Terms - circular/noncircular, proper/improper, rectilinear signal, coherence matrix, canonical correlations, circularity spectrum

\section{INTRODUCTION}

Recently, there has been an increased awareness that significant performance gains can be achieved by taking the information contained in the complementary covariance [1] matrix $\mathbf{R}_{z}^{\prime}=\mathrm{E}\left(\mathbf{z z}^{T}\right)$ (termed as relation matrix in [2], pseudo covariance matrix in [3] and second covariance in [4]) into account in second-order algorithms previously based on the standard covariance matrix $\mathbf{R}_{z}=\mathrm{E}\left(\mathbf{z z}^{H}\right)$ only (see, e.g., [5]). In the past, it was often assumed that $\mathbf{R}_{z}^{\prime}=\mathbf{0}$, a case that is referred to as either proper, second-order circular or circularly symmetric. However in digital communications, modulated signals may be improper or second-order non-circular but not necessarily with a maximum non-circularity rate, i.e., rectilinear as it has been often considered in the literature (e.g., in direction of arrival estimation, [6, 7]). For example, binary phase shift keying (BPSK) modulation is rectilinear in contrast to Gaussian minimum shift keying (GMSK) modulation which is improper but not rectilinear after derotation.

This paper addresses the measure of the degree of secondorder non-circularity or impropriety of complex random variables which can be used to come up with appropriate algorithms or to assess detection or estimation performances of algorithms adapted to improper signals. Its purpose is to complement previously available theoretical results $[1,2,3,8$, 9]. New properties of the canonical correlation between $\mathbf{z}$ and $\mathbf{z}^{*}$ (also called non-circularity rate in [4] and circular- ity spectrum in [3]) and of the augmented covariance matrice $\mathbf{R}_{\tilde{z}}=\mathrm{E}\left(\tilde{\mathbf{z}} \tilde{\mathbf{z}}^{H}\right)$ with $\tilde{\mathbf{z}} \stackrel{\text { def }}{=}\left(\mathbf{z}^{T}, \mathbf{z}^{H}\right)^{T}$ are given for scalar and multidimensional complex random variables with a particular attention paid to rectilinear random variables, i.e., with maximum circularity spectrum. Finally, maximum likelihood (ML) estimate of the circularity spectrum in the Gaussian case and asymptotic distribution of this estimate for arbitrary distributions are given.

The paper is organized as follows. Section 2 is dedicated to scalar complex random variables, while, Section 3 extends these results to multidimensional complex random variables.

\section{SCALAR COMPLEX RANDOM VARIABLE}

Let $z=x+i y$ denote a zero-mean second-order scalar complex random variable with variance $\sigma_{z}^{2} \stackrel{\text { def }}{=} \mathrm{E}\left|z^{2}\right|$ and complementary variance $\mathrm{E}\left(z^{2}\right)$. The non-circularity rate $\rho \in[0,1]$ and the non-circularity phase $\phi \in[0, \pi[$ of $z$ are defined by

$$
\rho e^{2 i \phi} \stackrel{\text { def }}{=} \frac{\mathrm{E}\left(z^{2}\right)}{\mathrm{E}\left|z^{2}\right|} .
$$

If $\rho=0, z$ is called proper [1] or circular to the second-order [2] and if $\rho=1, z$ is called rectilinear [10] because in this case $z=|z| e^{i \phi}$ and $z$ lies in one line of $\mathcal{C}$. If $\rho_{c o} \stackrel{\text { def }}{=} \frac{\mathrm{E}(x y)}{\sigma_{x} \sigma_{y}}$ with $\sigma_{x} \stackrel{\text { def }}{=} \sqrt{\mathrm{E}\left(x^{2}\right)}$ and $\sigma_{y} \stackrel{\text { def }}{=} \sqrt{\mathrm{E}\left(y^{2}\right)}$, denotes the correlation coefficient between the real $x$ and imaginary $y$ parts of $z$, we prove the following relations between $\rho$ and $\rho_{c o}$

Result 1 The non-circularity rate $\rho$ of a scalar complex random variable $z$ and the correlation coefficient $\rho_{\text {co }}$ between its real $x$ and imaginary $y$ parts are related by the following relations

- $\rho=1 \Leftrightarrow \rho_{c o}=1$,

- $\rho=0 \Rightarrow \rho_{c o}=0$, the converse is false because $\rho_{c o}=$ 0 does not imply $\sigma_{x}=\sigma_{y}$,

- $\rho \leq \rho_{c o}$ and $\rho=\rho_{c o}$ when $\sigma_{x}=\sigma_{y}$.

Proof These relations are straightforwardly deduced from the following expression of the non-circularity rate:

$$
\rho=\sqrt{\frac{\left(\frac{\sigma_{x}}{\sigma_{y}}-\frac{\sigma_{y}}{\sigma_{x}}\right)^{2}}{\left(\frac{\sigma_{x}}{\sigma_{y}}+\frac{\sigma_{y}}{\sigma_{x}}\right)^{2}}+4 \rho_{c o}^{2} \frac{1}{\left(\frac{\sigma_{x}}{\sigma_{y}}+\frac{\sigma_{y}}{\sigma_{x}}\right)^{2}} .}
$$


To interpret the non-circularity phase $\phi$ of $z$, we prove the following result

Result 2 For a non-circular scalar complex random variable $z$, the orthogonal regression line of the couple $(x, y)$ has a direction given by the non-circularity phase $\phi$ and the mean square orthogonal distance to this line is given ${ }^{1}$ by $\mathrm{E}\left(d^{2}\right)=$ $\frac{\sigma_{z}^{2}}{2}(1-\rho)$.

Proof The orthogonal regression line (see e.g., [11]) of the couple $(x, y)$ is given by the line orthogonal to the eigenvector $\mathbf{u}$ associated with the minimum eigenvalue $\lambda$ of the covariance matrix $\mathbf{R}_{w}$ of $\mathbf{w} \stackrel{\text { def }}{=}\left(\begin{array}{l}x \\ y\end{array}\right)$ and the mean square orthogonal distance $\mathrm{E}\left(d^{2}\right)$ to this line is given by $\lambda$.

To solve easily this problem, it is convenient to work with the augmented vector $\tilde{\mathbf{z}} \stackrel{\text { def }}{=}\left(\begin{array}{c}z \\ z^{*}\end{array}\right)$ whose covariance matrix $\mathbf{R}_{\tilde{z}}$ is related to $\mathbf{R}_{w}$ by $\mathbf{R}_{w}=\frac{1}{2} \mathbf{T}^{H} \mathbf{R}_{\tilde{z}} \mathbf{T}$ using $\tilde{\mathbf{z}}=$ $\sqrt{2} \mathbf{T w}$, where $\mathbf{T}$ is the unitary matrix $\frac{1}{\sqrt{2}}\left(\begin{array}{cc}1 & i \\ 1 & -i\end{array}\right)$. Because the minimum eigenvalue and the associated unit eigenvector of $\mathbf{R}_{\tilde{z}}=\sigma_{z}^{2}\left(\begin{array}{cc}1 & \rho e^{2 i \phi} \\ \rho e^{-2 i \phi} & 1\end{array}\right)$ are $\lambda=\sigma_{z}^{2}(1-$ $\rho)$ and $\mathbf{u}=\frac{i}{\sqrt{2}}\left(\begin{array}{c}e^{i \phi} \\ -e^{-i \phi}\end{array}\right)$, the minimum eigenvalue and the associated unit eigenvector of $\mathbf{R}_{w}$ are $\frac{1}{2} \lambda$ and $\mathbf{T}^{H} \mathbf{u}=$ $\left(\begin{array}{c}-\sin \phi \\ \cos \phi\end{array}\right) \perp\left(\begin{array}{c}\cos \phi \\ \sin \phi\end{array}\right)$.

Consequently, the larger is $\rho$, the smaller is the mean square distance of $(x, y)$ to the orthogonal regression line and this distance is zero if and only if $z$ is rectilinear along this orthogonal regression line whose direction is given by the noncircularity phase $\phi$.

Now, let us consider the estimation of the non-circularity rate $\rho$ from $T$ independent identically distributed realizations $\left(z_{t}\right)_{t=1, . ., T}$ for which the following result is proved in Appendix A.

Result 3 When $z_{t}$ is Gaussian distributed, the maximum likelihood $(M L)$ estimate $\left(\rho_{T}, \phi_{T}\right)$ of $(\rho, \phi)$ is given by $\left(\frac{\left|\sum_{t=1}^{T} z_{t}^{2}\right|}{\sum_{t=1}^{T}\left|z_{t}^{2}\right|}\right.$, $\left.\frac{1}{2} \operatorname{Arg}\left(\frac{\sum_{t=1}^{T} z_{t}^{2}}{\sum_{t=1}^{T}\left|z_{t}^{2}\right|}\right)\right)$. Furthermore, when $z_{t}$ is arbitrarily distributed, the sequence $\sqrt{T}\left(\rho_{T}-\rho\right)$ converges in distribution to the zero-mean Gaussian distribution of variance

$$
c_{\rho}=1-2 \rho^{2}+\rho^{4}+\rho^{2} \kappa+\frac{\kappa}{2}+\frac{\rho^{2} \Re\left(\kappa^{\prime}\right)}{2}-2 \rho^{2} \Re\left(\kappa^{\prime \prime}\right)
$$

where $\kappa, \kappa^{\prime}$ and $\kappa^{\prime \prime}$ are the normalized-like cumulants $\frac{\operatorname{Cum}\left(z, z, z^{*}, z^{*}\right)}{\left(\mathrm{E}\left(|z|^{2}\right)\right)^{2}}, \frac{\operatorname{Cum}(z, z, z, z)}{\left(\mathrm{E}\left(z^{2}\right)\right)^{2}}$ and $\frac{\operatorname{Cum}\left(z, z, z, z^{*}\right)}{\mathrm{E}\left(|z|^{2}\right) \mathrm{E}\left(z^{2}\right)}$ respectively.

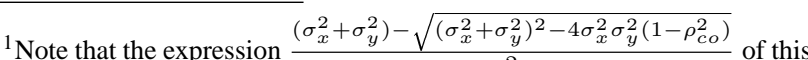
distance as a function of the correlation coefficient $\rho_{\text {co }}$ given by the minimum eigenvalue of $\mathbf{R}_{w}$ is much involved.
}

Note that the covariance of the asymptotic distribution of $\rho_{T}$ is a decreasing function of $\rho$ when $z_{t}$ is Gaussian distributed $\left(\kappa=\kappa^{\prime}=\kappa^{\prime \prime}=0\right)$ and vanishes for rectilinear random variables. Furthermore, using a derotation made by the normalized-like cumulants, the covariance of this empirical estimate does not depend of the non-circularity phase $\phi$ for arbitrary distributions.

\section{MULTIDIMENSIONAL COMPLEX RANDOM VARIABLE}

Consider now a full $K$-dimensional zero-mean second-order complex random variable $\mathbf{z}$. The canonical correlations between $\mathbf{z}$ and $\mathbf{z}^{*}$ i.e., the circularity spectrum of $\mathbf{z}$, are denoted by $\left(\rho_{k}\right)_{k=1, \ldots, K}$ and are arranged in decreasing order $1=\rho_{1}=\ldots=\rho_{r}>\rho_{r+1} \geq \ldots \geq \rho_{K} \geq 0$. Let $\mathbf{R}_{\tilde{z}} \stackrel{\text { def }}{=} \mathrm{E}\left(\tilde{\mathbf{z}} \tilde{\mathbf{z}}^{H}\right)=\left(\begin{array}{cc}\mathbf{R}_{z} & \mathbf{R}_{z}^{\prime} \\ \mathbf{R}_{z}^{\prime *} & \mathbf{R}_{z}^{*}\end{array}\right)$ denote the covariance matrix of the augmented vector $\tilde{\mathbf{z}} \stackrel{\text { def }}{=}\left(\begin{array}{c}\mathbf{z} \\ \mathbf{z}^{*}\end{array}\right)$ where $\mathbf{R}_{z}$ is nonsingular, and $\mathbf{R}_{w} \stackrel{\text { def }}{=} \mathrm{E}\left(\mathbf{w w}^{T}\right)=\left(\begin{array}{cc}\mathbf{R}_{x} & \mathbf{R}_{x, y} \\ \mathbf{R}_{y, x} & \mathbf{R}_{y}\end{array}\right)$, those of $\mathbf{w}=\left(\begin{array}{l}\mathbf{x} \\ \mathbf{y}\end{array}\right)$. Regarding the rank of these covariance matrices, the following result is proved

Result 4 For a full $K$-dimensional random variable $\mathbf{z}$, the rank of the covariance matrices $\mathbf{R}_{\tilde{z}}$ and $\mathbf{R}_{w}$ are equal to $2 K-r$ with $r \in\{0, . ., K\}$.

Proof As for the scalar case, $\mathbf{R}_{\tilde{z}}$ and $\mathbf{R}_{w}$ are related by $\mathbf{R}_{w}=$ $\frac{1}{2} \mathbf{T}^{H} \mathbf{R}_{\tilde{z}} \mathbf{T}$ where $\mathbf{T}$ is the unitary matrix $\frac{1}{\sqrt{2}}\left(\begin{array}{cc}\mathbf{I} & i \mathbf{I} \\ \mathbf{I} & -i \mathbf{I}\end{array}\right)$, consequently $\operatorname{rank}\left(\mathbf{R}_{\tilde{z}}\right)=\operatorname{rank}\left(\mathbf{R}_{w}\right)$. Now consider $\mathbf{R}_{\tilde{z}}$. From the definition of the coherence matrix ${ }^{2}$

$$
\mathbf{M}=\mathbf{R}_{z}^{-1 / 2} \mathbf{R}_{z}^{\prime} \mathbf{R}_{z}^{-T / 2}
$$

associated with $\mathbf{z}$ and $\tilde{\mathbf{z}}, \mathbf{R}_{\tilde{z}}$ may be factored (see e.g., [8]) as

$\mathbf{R}_{\tilde{z}}=\left(\begin{array}{cc}\mathbf{R}_{z}^{1 / 2} & \mathbf{O} \\ \mathbf{O} & \mathbf{R}_{z}^{* / 2}\end{array}\right)\left(\begin{array}{cc}\mathbf{I} & \mathbf{M} \\ \mathbf{M}^{*} & \mathbf{I}\end{array}\right)\left(\begin{array}{cc}\mathbf{R}_{z}^{H / 2} & \mathbf{O} \\ \mathbf{O} & \mathbf{R}_{z}^{T / 2}\end{array}\right)$

Since $\mathbf{M}$ is complex symmetric, there exists a specular singular value decomposition (SVD), called Takagi's factorization, which is $\mathbf{M}=\mathbf{U} \boldsymbol{\Delta} \mathbf{U}^{T}$, where $\boldsymbol{\Delta}=\operatorname{Diag}\left(\rho_{1}, \ldots, \rho_{K}\right)$ and $\mathbf{U}$ is a unitary matrix. Combining this decomposition of $\mathbf{M}$ in

\footnotetext{
${ }^{2}$ Note that the coherence matrix $\mathbf{M}$ depends on the specific square root $\mathbf{R}_{z}^{1 / 2}$ of $\mathbf{R}_{z}$, unique only if it is imposed to be positive definite Hermitian, in contrast to the circularity spectrum $\left(\rho_{1}, \ldots, \rho_{K}\right)$ which is always unique $[3$, th.2].
} 
the previous expression of $\mathbf{R}_{\tilde{z}}$, we obtain

$$
\begin{aligned}
\mathbf{R}_{\tilde{z}}= & \left(\begin{array}{cc}
\mathbf{R}_{z}^{1 / 2} & \mathbf{O} \\
\mathbf{O} & \mathbf{R}_{z}^{* / 2}
\end{array}\right)\left(\begin{array}{cc}
\mathbf{U} & \mathbf{O} \\
\mathbf{O} & \mathbf{U}^{*}
\end{array}\right)\left(\begin{array}{cc}
\mathbf{I} & \boldsymbol{\Delta} \\
\boldsymbol{\Delta} & \mathbf{I}
\end{array}\right) \\
& \left(\begin{array}{cc}
\mathbf{U}^{H} & \mathbf{O} \\
\mathbf{O} & \mathbf{U}^{T}
\end{array}\right)\left(\begin{array}{cc}
\mathbf{R}_{z}^{H / 2} & \mathbf{O} \\
\mathbf{O} & \mathbf{R}_{z}^{T / 2}
\end{array}\right)
\end{aligned}
$$

Consequently $\operatorname{rank}\left(\mathbf{R}_{\tilde{z}}\right)=\operatorname{rank}\left(\begin{array}{cc}\mathbf{I} & \boldsymbol{\Delta} \\ \boldsymbol{\Delta} & \mathbf{I}\end{array}\right)=\operatorname{rank}(\mathbf{I})+$ $\operatorname{rank}\left(\mathbf{I}-\boldsymbol{\Delta} \mathbf{I}^{-1} \boldsymbol{\Delta}\right)$ using [12, th.8.5.10] that gives the rank of a partitioned matrix. So $\operatorname{rank}\left(\mathbf{R}_{\tilde{z}}\right)=K+\operatorname{rank}\left(\mathbf{I}-\boldsymbol{\Delta}^{2}\right)=$ $K+(K-r)$.

Regarding the maximum of the circularity spectrum, the following equivalence is proved

Result 5 The circularity spectrum is maximum, i.e., $\rho_{1}=$ $\rho_{2}=\ldots=\rho_{K}=1$ if and only if $(i) \operatorname{rank}\left(\mathbf{R}_{\tilde{z}}\right)=K$ (i.e., $\tilde{\mathbf{z}}$ belongs to a $K$-dimensional subspace of $\mathcal{C}^{2 K}$ ), (ii) $\operatorname{rank}\left(\mathbf{R}_{w}\right)=K$ (i.e., w belongs to a $K$-dimensional subspace of $\mathcal{R}^{2 K}$ ), (iii) there exists a square root $\mathbf{R}_{z}^{1 / 2}$ of $\mathbf{R}_{z}$ such that $\mathbf{R}_{z}^{\prime}=\mathbf{R}_{z}^{1 / 2} \mathbf{R}_{z}^{* / 2}$, (iv) there exists square roots $\mathbf{R}_{x}^{1 / 2}$ and $\mathbf{R}_{y}^{1 / 2}$ of $\mathbf{R}_{x}$ and $\mathbf{R}_{y}$ respectively, such that $\mathbf{R}_{x, y}=$ $\mathbf{R}_{x}^{1 / 2} \mathbf{R}_{y}^{1 / 2}$.

Proof The equivalences (i) and (ii) are a direct consequence of $\operatorname{rank}\left(\mathbf{R}_{\tilde{z}}\right)=\operatorname{rank}\left(\mathbf{R}_{w}\right)=K+\operatorname{rank}\left(\mathbf{I}-\boldsymbol{\Delta}^{2}\right)$. If the circularity spectrum is maximum, $\boldsymbol{\Delta}=\mathbf{I}$ and (iii) follows because (2) implies $\mathbf{R}_{z}^{\prime}=\mathbf{R}_{z}^{1 / 2} \mathbf{U} \mathbf{U}^{T} \mathbf{R}_{z}^{T / 2}$ where $\mathbf{R}_{z}^{1 / 2} \mathbf{U}$ is a square root of $\mathbf{R}_{z}$. Conversely, (iii) implies that

$$
\mathbf{R}_{\tilde{z}}=\left(\begin{array}{l}
\mathbf{R}_{z}^{1 / 2} \\
\mathbf{R}_{z}^{* / 2}
\end{array}\right)\left(\begin{array}{ll}
\mathbf{R}_{z}^{1 / 2} & \mathbf{R}_{z}^{* / 2}
\end{array}\right)
$$

which involves that $\operatorname{rank}\left(\mathbf{R}_{\tilde{z}}\right)=K$ and the circularity spectrum is maximum. Equivalence (iv) follows the same lines that equivalence (iii) by considering the canonical correlations associated with $\mathbf{x}$ and $\mathbf{y}$ and equivalence (ii).

By analogy with the scalar case, we propose to call rectilinear such complex multidimensional random variables $\mathbf{z}$ whose circularity spectrum is maximum. Note that if the components $\left(z_{1}, \ldots, z_{K}\right)$ of $\mathbf{z}$ are all rectilinear, there are $K$ linear relations $y_{k}=\tan \left(\phi_{z_{k}}\right) x_{k},(k=1, . ., K)$ between the components of $\mathbf{w}$, consequently $\operatorname{rank}\left(\mathbf{R}_{w}\right)=K$ and $\mathbf{z}$ is rectilinear $^{3}$. But the converse is not true: if $\mathbf{z}$ is rectilinear, its components $\left(z_{k}\right)_{k=1, \ldots, K}$ need not have maximum non-circular rates $\rho_{z_{k}}$. For example, let $\mathbf{z}=\left(z_{1}, z_{2}\right)^{T}$ where $z_{1}$ is circular and $z_{2}=x_{2}+i y_{2}$ with $x_{2}=a x_{1}$ and $y_{2}=a y_{1} . \mathbf{z}$ is rectilinear because $\mathbf{w}$ belongs to a 2-dimensional subspace of $\mathcal{R}^{4}$ but the non-circularity rates of $z_{1}$ and $z_{2}$ are $\rho_{z_{1}}=0$ and $\rho_{z_{2}}=\frac{\left|a^{2}-1\right|}{a^{2}+1}$ with $\rho_{z_{2}}=0$ for $a=1$.

\footnotetext{
${ }^{3}$ Note that the components $\left(z_{k}\right)_{k=1, \ldots, K}$ of $\mathbf{z}$ do not need to be uncorrelated as it is usually assumed in DOA estimation of non-circular sources (see e.g. $[6,7])$.
}

To extend to the multidimensional case, the non-circularity phase $\phi$ defined in the scalar case by (1), we propose a definition based on the $K$-dimensional orthogonal regression subspace of $\left(x_{1}, \ldots, x_{K}, y_{1}, \ldots, y_{K}\right)$ which is the support of $\mathbf{w}$ for a maximum circularity spectrum. The canonical angles $\left(\phi_{1}, \phi_{2}, . ., \phi_{K^{2}}\right)$ between this subspace and each of the $K$ hyperspaces $\left(y_{k}=0\right)_{k=1, \ldots, K}$ of $\mathcal{R}^{2 K}$ satisfy this aim. However, two questions remain open. First, does one extend the expression of the mean square orthogonal distance to this $K$ dimensional orthogonal regression subspace given in Result 2 ? Second, does one prove that the parameter $\left(\boldsymbol{\rho}, \phi, \mathbf{R}_{z}\right)$ with $\phi \stackrel{\text { def }}{=}\left(\phi_{1}, \phi_{2}, . ., \phi_{K^{2}}\right)^{T}$ makes up a one to one parametrization of $\left(\mathbf{R}_{z}, \mathbf{R}_{z}^{\prime}\right)$ ?

Now, let us consider the estimation of the circularity spectrum $\boldsymbol{\rho}=\left(\rho_{1}, \rho_{2}, \ldots, \rho_{K}\right)^{T}$ from $T$ independent identically distributed realizations $\left(\mathbf{z}_{t}\right)_{t=1, \ldots, T}$ for which the following result is proved in [14] using the same steps that for Result 3.

Result 6 When $\mathbf{z}_{t}$ is Gaussian distributed, the ML estimate $\boldsymbol{\rho}_{T}$ of $\boldsymbol{\rho}$ is given by the vector containing the $K$ singular values of the empirical coherence matrix

$$
\mathbf{M}_{T}=\mathbf{R}_{z, T}^{-1 / 2} \mathbf{R}_{z, T}^{\prime} \mathbf{R}_{z, T}^{-T / 2}
$$

where $\mathbf{R}_{z, T} \stackrel{\text { def }}{=} \frac{1}{T} \sum_{t=1}^{T} \mathbf{z}_{t} \mathbf{z}_{t}^{H}$ and $\mathbf{R}_{z, T}^{\prime} \stackrel{\text { def }}{=} \frac{1}{T} \sum_{t=1}^{T} \mathbf{z}_{t} \mathbf{z}_{t}^{T}$. Furthermore, when $\mathbf{z}_{t}$ is arbitrarily distributed and when the circularity spectrum $\rho$ has distinct elements, the sequence $\sqrt{T}\left(\boldsymbol{\rho}_{T}-\boldsymbol{\rho}\right)$ converges in distribution to a zero-mean Gaussian distribution that extends Result 3, whose covariance is specified in [14].

\section{A. APPENDIX: PROOF OF RESULT 3}

When $z_{t}$ is Gaussian distributed, the log-likelihood function associated with $\left(\mathbf{z}_{t}\right)_{t=1, \ldots, T}$ can be classically written after dropping the constants as

$$
L\left(\rho, \phi, \sigma_{z}^{2}\right)=-\frac{T}{2}\left(\ln \left[\operatorname{Det}\left(\mathbf{R}_{\tilde{z}}\right)\right]+\operatorname{Tr}\left(\mathbf{R}_{\tilde{z}}^{-1} \mathbf{R}_{\tilde{z}, T}\right)\right)
$$

with $\mathbf{R}_{\tilde{z}, T} \stackrel{\text { def }}{=} \frac{1}{T} \sum_{t=1}^{T} \tilde{\mathbf{z}}_{t} \tilde{\mathbf{z}}_{t}^{H}$ where the parameter $\left(\rho, \phi, \sigma_{z}^{2}\right)$ is embedded in the covariance matrix $\mathbf{R}_{\tilde{z}}$. Due to the structure $\left[\begin{array}{cc}(\times) & (\diamond)^{*} \\ (\diamond)^{*} & (\times)^{*}\end{array}\right]$ of $\mathbf{R}_{\tilde{z}}$ the ML estimation of $\mathbf{R}_{\tilde{z}}$ becomes a constrained optimization problem which is not standard. But maximizing the log-likelihood (3) without any constraint on the Hermitian matrix $\mathbf{R}_{\tilde{z}}$ reduces to a standard maximization problem, whose solution is $\mathbf{R}_{\tilde{z}, T}$. Because

$$
\mathbf{R}_{\tilde{z}, T}=\left[\begin{array}{cc}
\frac{1}{T} \sum_{t=1}^{T}\left|z_{t}^{2}\right| & \frac{1}{T} \sum_{t=1}^{T} z_{t}^{2} \\
\frac{1}{T} \sum_{t=1}^{T} z_{t}^{* 2} & \frac{1}{T} \sum_{t=1}^{T}\left|z_{t}^{2}\right|
\end{array}\right]
$$

is also structured as $\left[\begin{array}{cc}(\times) & (\diamond) \\ (\diamond)^{*} & (\times)^{*}\end{array}\right], \mathbf{R}_{\tilde{z}, T}$ is the ML estimate of $\mathbf{R}_{\tilde{z}}$. Using the invariance property of the ML estimate 
implies that the ML estimate of $(\rho, \phi)$ is given by

$$
\left(\frac{\left|\sum_{t=1}^{T} z_{t}^{2}\right|}{\sum_{t=1}^{T}\left|z_{t}^{2}\right|}, \frac{1}{2} \operatorname{Arg}\left(\frac{\sum_{t=1}^{T} z_{t}^{2}}{\sum_{t=1}^{T}\left|z_{t}^{2}\right|}\right)\right) .
$$

Deriving the asymptotic distribution of the empirical estimate $\rho_{T}$ when $z_{t}$ is arbitrarily distributed, relies on the standard central limit theorem ${ }^{4}$ applied to the independent identically distributed bidimensional complex random variables $\left(\begin{array}{c}r_{z, T} \\ r_{z, T}^{\prime}\end{array}\right)$ with $r_{z, T}=\frac{1}{T} \sum_{t=1}^{T}\left|z_{t}^{2}\right|$ and $r_{z, T}^{\prime}=\frac{1}{T} \sum_{t=1}^{T} z_{t}^{2}$ :

$\sqrt{T}\left(\begin{array}{c}r_{z, T}-r_{z} \\ r_{z, T}^{\prime}-r_{z}^{\prime}\end{array}\right)$

$$
\stackrel{\mathcal{L}}{\rightarrow} \mathcal{N}_{C}\left(\left(\begin{array}{l}
0 \\
0
\end{array}\right),\left(\begin{array}{cc}
c_{r} & c_{r, r^{\prime}} \\
c_{r^{\prime}, r} & c_{r^{\prime}}
\end{array}\right),\left(\begin{array}{cc}
c_{r}^{\prime} & c_{r, r^{\prime}}^{\prime} \\
c_{r^{\prime}, r}^{\prime} & c_{r^{\prime}}^{\prime}
\end{array}\right)\right),
$$

where $r_{z}=\mathrm{E}\left|z_{t}^{2}\right|=\sigma_{z}^{2}$ and $r_{z}^{\prime}=\mathrm{E}\left(z_{t}^{2}\right)=\rho \sigma_{z}^{2} e^{i 2 \phi}$. Using the identity

$$
\begin{aligned}
& \mathrm{E}\left(z_{1} z_{2} z_{3} z_{4}\right)=\operatorname{Cum}\left(z_{1}, z_{2}, z_{3}, z_{4}\right) \\
& \quad+\mathrm{E}\left(z_{1} z_{2}\right) \mathrm{E}\left(z_{3} z_{4}\right)+\mathrm{E}\left(z_{1} z_{3}\right) \mathrm{E}\left(z_{2} z_{4}\right)+\mathrm{E}\left(z_{1} z_{4}\right) \mathrm{E}\left(z_{2} z_{3}\right),
\end{aligned}
$$

we obtain

$$
\begin{aligned}
&\left(\begin{array}{cc}
c_{r} & c_{r, r^{\prime}} \\
c_{r^{\prime}, r} & c_{r^{\prime}}
\end{array}\right)=\sigma_{z}^{4}\left(\begin{array}{cc}
1+\rho^{2}+\kappa & \rho e^{-i 2 \phi}\left(2+\kappa^{\prime \prime *}\right) \\
\rho e^{i 2 \phi}\left(2+\kappa^{\prime \prime}\right) & 2+\kappa
\end{array}\right) \\
&\left(\begin{array}{cc}
c_{r}^{\prime} & c_{r, r^{\prime}}^{\prime} \\
c_{r^{\prime}, r}^{\prime} & c_{r^{\prime}}^{\prime}
\end{array}\right)=\sigma_{z}^{4}\left(\begin{array}{cc}
1+\rho^{2}+\kappa & \rho e^{i 2 \phi}\left(2+\kappa^{\prime \prime}\right) \\
\rho e^{i 2 \phi}\left(2+\kappa^{\prime \prime}\right) & \rho^{2} e^{i 4 \phi}\left(2+\kappa^{\prime}\right)
\end{array}\right) .
\end{aligned}
$$

Then, considering the following mappings

$$
\left(r_{z, T}, r_{z, T}^{\prime}\right) \longmapsto m_{T}=\frac{r_{z, T}^{\prime}}{r_{z, T}} \longmapsto \rho_{T}=\sqrt{m_{T} m_{T}^{*}}
$$

with their associated differentials

$d m=-\frac{r^{\prime}}{r^{2}} d r+\frac{1}{r} d r^{\prime} \quad$ and $\quad d \rho=\frac{1}{2 \rho}\left(m^{*} d m+m d m^{*}\right)$, the standard theorem of continuity (see e.g., [13, p. 122]) on regular functions of asymptotically Gaussian statistics applies. Consequently, we have with $m=\frac{r_{z}^{\prime}}{r_{z}}=\rho e^{i 2 \phi}$

$$
\sqrt{T}\left(m_{T}-m\right) \stackrel{\mathcal{L}}{\rightarrow} \mathcal{N}_{C}\left(0, c_{m}, c_{m}^{\prime}\right)
$$

where

$$
\begin{aligned}
& c_{m}=\left(\begin{array}{ll}
-\frac{r_{z}^{\prime}}{r_{z}^{2}} & \frac{1}{r_{z}}
\end{array}\right)\left(\begin{array}{cc}
c_{r} & c_{r, r^{\prime}} \\
c_{r^{\prime}, r} & c_{r^{\prime}}
\end{array}\right)\left(\begin{array}{c}
-\frac{r_{z}^{\prime *}}{r_{z}^{2}} \\
\frac{1}{r_{z}}
\end{array}\right), \\
& c_{m}^{\prime}=\left(\begin{array}{cc}
-\frac{r_{z}^{\prime}}{r_{z}^{2}} & \frac{1}{r_{z}}
\end{array}\right)\left(\begin{array}{cc}
c_{r}^{\prime} & c_{r, r^{\prime}}^{\prime} \\
c_{r^{\prime}, r}^{\prime} & c_{r^{\prime}}^{\prime}
\end{array}\right)\left(\begin{array}{c}
-\frac{r_{z}^{\prime}}{r_{z}^{2}} \\
\frac{1}{r_{z}}
\end{array}\right)
\end{aligned}
$$

${ }^{4} \mathcal{N}_{R}(\mathbf{m}, \mathbf{C})$ and $\mathcal{N}_{C}\left(\mathbf{m}, \mathbf{C}, \mathbf{C}^{\prime}\right)$ denote Gaussian real and complex distribution with mean, covariance and complementary covariance are $\mathbf{m}, \mathbf{C}$ and $\mathbf{C}^{\prime}$ respectively. and

$$
\sqrt{T}\left(\rho_{T}-\rho\right) \stackrel{\mathcal{L}}{\rightarrow} \mathcal{N}_{R}\left(0, c_{\rho}\right),
$$

where $c_{\rho}=\frac{1}{4 \rho^{2}}\left(\begin{array}{ll}m^{*} & m\end{array}\right)\left(\begin{array}{cc}c_{m} & c_{m}^{\prime} \\ c_{m}^{\prime}{ }^{*} & c_{m}{ }^{*}\end{array}\right)\left(\begin{array}{c}m \\ m^{*}\end{array}\right)=$ $\frac{1}{2}\left(c_{m}+\Re\left(c_{m}^{\prime} e^{-4 i \phi}\right)\right)$. Result 3 follows thanks to simple algebraic manipulations of $c_{\rho}$.

\section{B. REFERENCES}

[1] P.J. Schreier and L. Scharf, "Second-order Analysis of improper complex random vectors and processes," IEEE Trans. on Signal Processing, vol. 51, no. 3, pp. 714-725, March 2003.

[2] B. Picinbono, "Second-order complex random vectors and normal distributions," IEEE Trans. on Signal Processing, vol. 44, no. 10, pp. 2637-2640, October 1996.

[3] J. Eriksson and V. Koivunen, "Complex random vectors and ICA models: identifiability, uniqueness, and separability," IEEE Trans. on Information Theory, vol. 52 no. 3, pp. 10171029, March 2006.

[4] H. Abeida and J.P. Delmas, "MUSIC-like estimation of direction of arrival for non-circular sources," IEEE Trans. on Signal Processing, vol. 54, no. 7, pp. 2678-2690, July 2006.

[5] P.J. Schreier, L. Scharf and C.T. Mullis, "Detection and estimation of improper complex random signals," IEEE Trans. on Information Theory, vol. 51, no. 1, pp. 306-312, January 2005.

[6] P. Chargé, Y. Wang and J. Saillard, "A non-circular sources direction finding method using polynomial rooting," Signal Processing, vol. 81, pp. 1765-1770, 2001.

[7] M. Haardt and F. Römer, "Enhancements of unitary ESPRIT for non circular sources," International Conference on ASSP, Montreal, May 2004.

[8] L. Scharf and C.T. Mullis, "Canonical cordinates and the geometry of inference rate and capacity," IEEE Trans. on Signal Processing, vol. 48, no. 3, pp. 824-831, March 2000.

[9] E.M. Cramer and W.A. Nicewander, "Some symmetricinvariant measures of multivariate association," Psychometrika, vol. 44, no. 1, pp. 43-54, March 1979.

[10] P. Chevalier and F. Pipon, "New insights into optimal widely linear array receivers for the demodulation of BPSK, MSK and GMSK signals corrupted by non circular interferences - Application to SAIC", IEEE Trans. on Signal Processing, vol.54, no.3, pp. 870-883, March 2006.

[11] I.T. Jolliffe, Principal Component Analysis Series: Springer Series in Statistics, 2002.

[12] D.A. Harville, Matrix algebra from a statistician's perspective, Springer 1999.

[13] R.J. Serfling, Approximation Theorems of Mathematical Statistics, John Wiley and Sons, 1980.

[14] J.P. Delmas and H. Abeida, "On the degree of second-order non-circularity of complex random variables", submitted to IEEE Trans. on Signal Processing. 